\title{
Does Incorporation of Silicon Increases Lipophilicity? A Theoretical Perspective
}

\author{
Lakhya J Mazumder, Chayanika Kashyap, Sabnam S Ullah and Ankur K Guha* \\ Department of Chemistry, Cotton University, India
}

Received: February 27, 2018; Published: March 08, 2018

*Corresponding author: Ankur K Guha, Department of Chemistry, Cotton University, Guwahati, Assam, India, Email: ankurkantiguha@gmail.com

\begin{abstract}
Quantum chemical calculations have been carried out using MP2/6-311++ $\mathrm{G}^{* *}$ level of theory to calculate lipophilicity, i.e., octanol-water partition coefficient ( $\log \mathrm{P})$ of some alcohols and their silicon derivatives. Very good correlation has been obtained between the experimental $\mathrm{LogP}$ values against our calculated one. Moreover, incorporation of $\mathrm{Si}$ atom into the $\mathrm{C}$ atom attached to the - $\mathrm{OH}$ group dramatically increases the lipophilicity of the compounds. This finding may have wide implications towards designing new Si incorporated drugs.
\end{abstract}

\section{Introduction}

The lipophilicity, which refers to the ability of a compound to dissolve in fats, oils, lipids and non polar solvents such as hexane or toluene, is an important parameter for a drug to successfully pass through clinical development [1-3]. The lipophilicity is a measure of all the intermolecular forces between solute and two phases between which it partitions [4]. Generally, the lipophilicity is expressed experimentally as the octanol-water partition coefficient $\mathrm{P}(\log \mathrm{P})$ or as distribution coefficients $(\log \mathrm{D})$. The $\log \mathrm{P}$ describes the partition equilibrium of an un-ionized solute, while $\log D$ determines the ratio of the sum of the concentrations of all forms of the compound ( $\mathrm{pH}$-dependent mixture of ionized and unionized forms) in each of the two phases. Accurate determination of LogP is very important requirement in drug design. Theoretical calculation of $\log \mathrm{P}$ has been practiced for a long time. In practice, theoretical LogP values evaluated using quantitative structure-activity relationship (QSAR) models are practiced more often rather than expensive experimental measurements [5-6]. However, screening based on QSAR models often lead to erroneous results [7-8].

Herein, we have undertaken a theoretical study on the direct and accurate measurement of $\log \mathrm{P}$ values based on the following equation

$$
\log P=\left(\Delta G_{\text {water }}-\Delta G_{n-\text { octanol }}\right) / R T
$$

Where values are the free energies in respective solvent [9], the calculated values of $\log \mathrm{P}$ were then compared with the available experimental data from Khadikar and co-workers [10]. Recently, it has been speculated that substitution of $\mathrm{C}$ atom by $\mathrm{Si}$ increases the lipophilicity of different drugs [11]. This is intriguing by the fact that $\mathrm{Si}$ is the biostere of $\mathrm{C}$ as $\mathrm{Si}$ has similar physicochemical properties and similar biological activity in broad sense. Herein, we are also interested in testing the speculation by carrying out high level abinitio calculations.

\section{Computational Details}

All these structures were fully optimized without any symmetry constraints at MP2/6-311++G** level of theory [12]. Harmonic frequency calculations were also carried out to understand the nature of the stationary states. All structures were found to be at the local minimum with all real frequencies. Solvent calculations were performed using the prescribed salvation model using density (SMD) approach. All calculations were performed using GAUSSIAN16 suite of program [13].

\section{Results and Discussions}

Figure 1 shows the optimized geometries of the molecules considered in this study. The geometries shown here are for the gas phase. All these structures are local minima on the potential energy surface. It should be noted that many isomeric forms of these structures are possible; however, we have considered the lowest energy isomer of all these molecules. These gas phase geometries are taken as the starting geometry for further geometry optimization in different solvent (water and n-octanol) medium using SMD approach. Table 1 contains the calculated values of $\log \mathrm{P}$ of the selected molecules. The experimental LogP values are taken from Khadikar and co-workers [10]. It is evident from 
Table 1 that the values calculated with MP2/6-311++G** level of theory lies between -0.3 to 3.3 and follows a similar trend as the experimental values. However, the magnitudes of calculated values are different from the experimental one. We, therefore, adopted regression analysis between the calculated and experimental LogP values. Figure 2 shows the plot of the regression analysis. It is evident from Figure 2 that there is a very good correlation between the calculated and experimental values with regression coefficient, $\mathrm{R}^{2}=0.97$. Interestingly, incorporation of $\mathrm{Si}$ atom into the alcohols dramatically increases the LogP value which is consistent with the recent speculation.

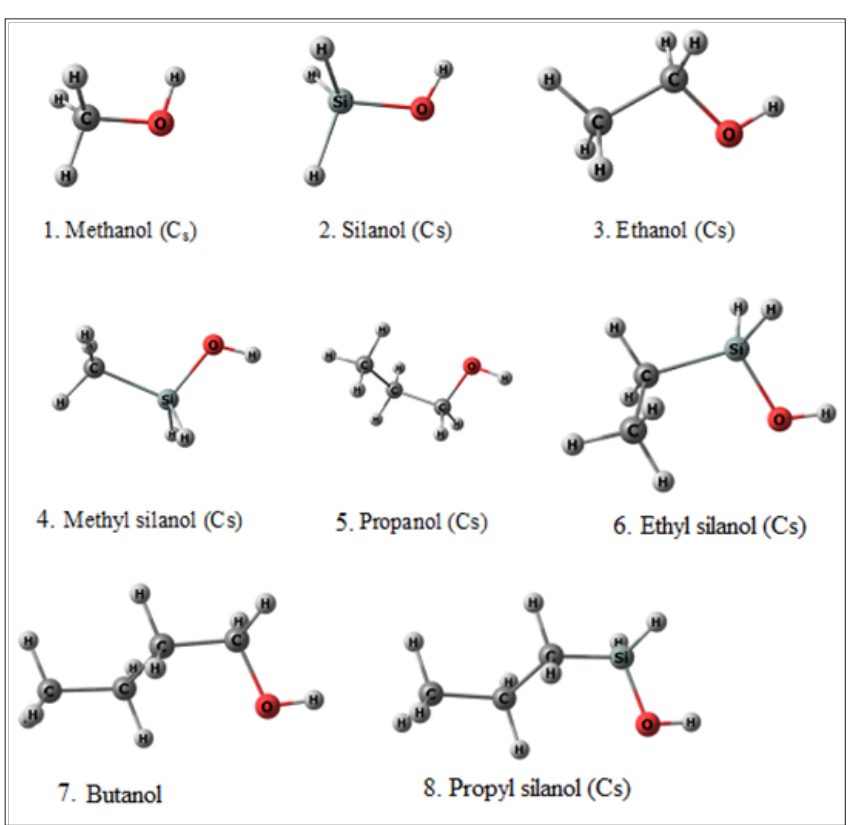

Figure 1: Local minimum structures of the investigated molecules calculated at MP2/6-311++G** level of theory.

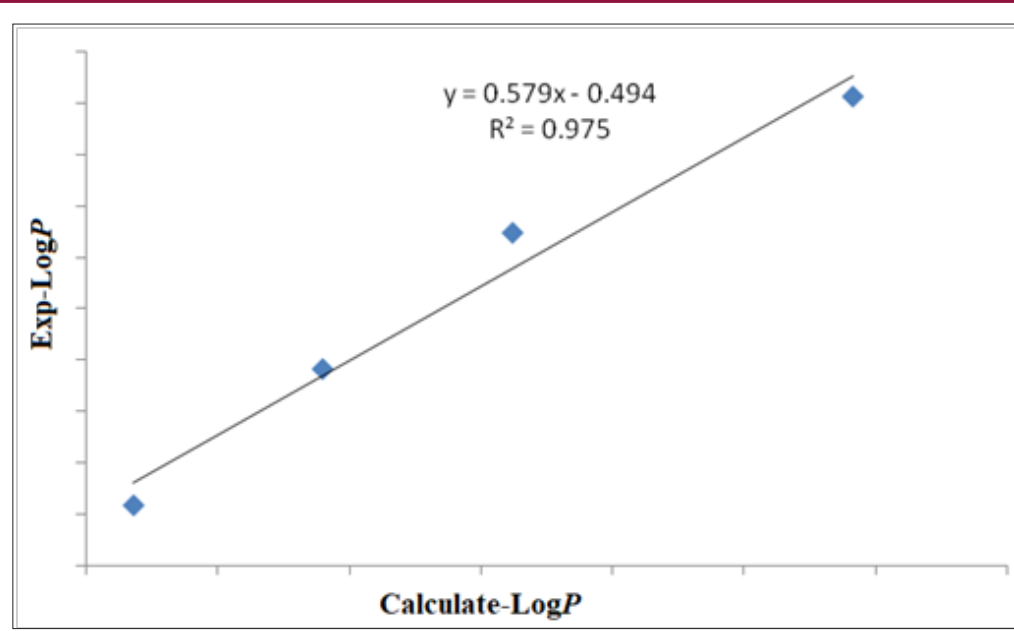

Figure 2: Correlation plot between the experimental and calculated LogP values.

Table 1: MP2/6-311++G** calculated values of $\log P$.

\begin{tabular}{|c|c|c|}
\hline \multirow{2}{*}{ Molecule } & LogP & \\
\cline { 2 - 3 } & Calculated & Experimental10 \\
\hline Methanol & -0.317 & -0.764 \\
\hline Silanol & 0.359 & \\
\hline Ethanol & 0.400 & -0.235 \\
\hline Methyl silanol & 0.805 & \\
\hline Propanol & 1.121 & 0.294 \\
\hline
\end{tabular}

\begin{tabular}{|c|l|l|}
\hline Ethyl silanol & 1.164 & \\
\hline Butanol & 2.412 & 0.823 \\
\hline Propyl silanol & 3.311 & \\
\hline
\end{tabular}

\section{Conclusion}

Abinitio calculations at MP2/6-311++G** level of theory has been carried out for the theoretical calculation of lipophilicity parameter LogP. A nice linear correlation has been obtained for the set of alcohols studied in this work. The most important outcome of 
this work is the theoretical verification of the previous speculation that incorporation of $\mathrm{Si}$ atom into the carbon containing drugs may increase the lipophilicity and hence, may increase the physiological activity of the Si containing drugs.

\section{Acknowledgement}

AKG thanks the Science and Engineering Research Board (SERB), Government of India for providing financial assistance in the form of a project (project no. ECR/2016/001466).

\section{References}

1. Lombardo F, Shalaeva MY, Tupper KA, Gao F, Abraham MH (2000) ElogPoct: a tool for lipophilicity determination in drug discovery. J Med Chem 43(15): 2922-2928.

2. Sârbu C, Naşcu-Briciu RD, Casoni D, Kot-Wasik A, Wasik A, et al. (2012) Chromatographic lipophilicity determination using large volume injections of the solvents non-miscible with the mobile phase. J Chromatogr A 1266: 53-60.

3. Komsta $七$, Skibiński R, Berecka A, Gumieniczek A, Radkiewicz B, et al. (2010) Revisiting thin-layer chromatography as a lipophilicity determination tool-a comparative study on several techniques with a model solute set. J Pharmaceut Biomed 53(4): 911-918.

4. (1997) IUPAC, Compendium of Chemical Terminology, ( $2^{\text {nd }}$ Edition) Blackwell Scientific Publications, Oxford.
5. (2016) Molinspiration Cheminformatics, Bratislava, Slovak Republic.

6. Estrada-Tejedor R, Sabaté N, Broto F, Nonell S (2013) the validation of quantum chemical lipophilicity prediction of alcohols. Afinidad 70: 564.

7. Liao SY, Chen JC, Qian L, Shen Y, Zheng KC (2008) QSAR, action mechanism and molecular design of flavone and isoflavone derivatives with cytotoxicity against HeLa. Eur J Med Chem 43(10): 2159-2170.

8. Khan AU (2016) Descriptors and their selection methods in QSAR analysis: paradigm for drug design. Drug Discov Today 21(18): 12911302.

9. Garrido NM, Economou IG, Queimada AJ, Jorge M, Macedo EA (2012) Prediction of the n-hexane/water and 1-octanol/water partition coefficients for environmentally relevant compounds using molecular simulation. AIChE J 58(6): 1929-1938.

10. Khadikar PV, Sharma V, Varma RG (2005) Novel estimation of lipophilicity using 13C NMR chemical shifts as molecular descriptor. Bioorg Med Chem Lett 15(2): 421-425.

11. Ramesh R, Reddy DS (2017) Quest for Novel Chemical Entities through Incorporation of Silicon in Drug Scaffolds. J Med Chem.

12. Møller C, Plesset MS (1934) Note on an Approximation Treatment for Many-Electron Systems. Physn Rev 46: 618-622.

13. Frisch MJ, Trucks GW, Schlegel HB, GE Scuseria GE, Robb MA, et al. Cioslowski J and Fox DJ Gaussian 16, Revision A.03, Gaussian, Inc. Wallingford CT.

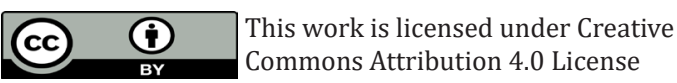

Submission Link: https://biomedres.us/submit-manuscript.php

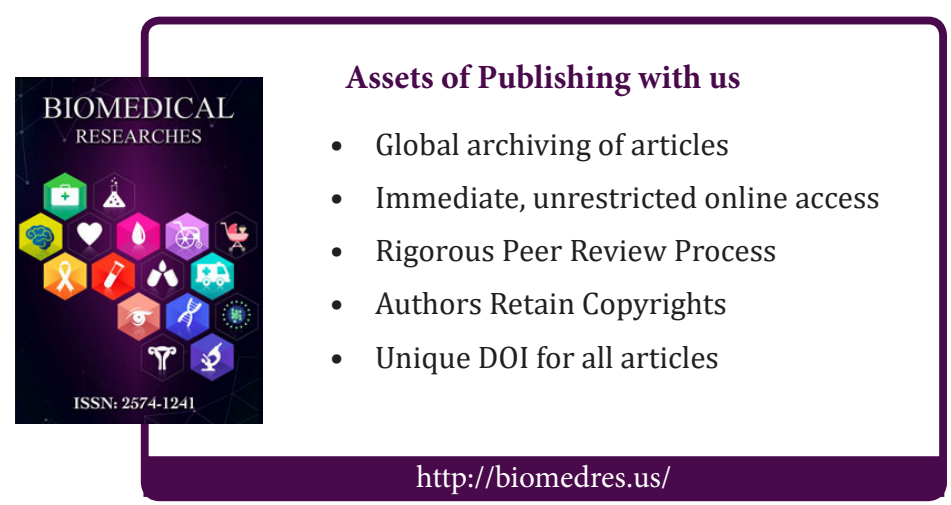

